
\title{
$\begin{array}{ll}\text { Research Square } & \begin{array}{l}\text { Preprints are preliminary reports that have not undergone peer review. } \\ \text { They should not be considered conclusive, used to inform clinical practice, } \\ \text { or referenced by the media as validated information. }\end{array}\end{array}$
}

\section{Development and validation of the isothermal recombinase polymerase amplification assays for rapid detection of Mycoplasma ovipneumoniae}

\author{
Jinfeng Wang \\ Technology Center of Shijiazhuang Customs Distrcit \\ Ruiwen Li \\ Hebei Agricultural University \\ Xiaoxia Sun \\ Technology center of Shijiazhuang Customs District \\ Libing Liu \\ Technology Center of Shijiazhuang Customs Distrcit \\ Xuepiao Hao \\ Hebei Agricultural University \\ Jianchang Wang \\ Technology Center of Shijiazhuang Customs District \\ Wanzhe Yuan ( $\nabla$ yuanwanzhe2015@126.com ) \\ College of Veterinary Medicine, Agricultural University of Hebei https://orcid.org/0000-0003-2442-0539
}

\section{Methodology article}

Keywords: Mycoplasma ovipneumoniae, 16S rRNA gene, real-time RPA, lateral flow strip, isothermal amplification

Posted Date: May 12th, 2020

DOI: https://doi.org/10.21203/rs.3.rs-17865/v2

License: () (1) This work is licensed under a Creative Commons Attribution 4.0 International License. Read Full License

Version of Record: A version of this preprint was published at BMC Veterinary Research on June 1st, 2020. See the published version at https://doi.org/10.1186/s12917-020-02387-3. 


\section{Abstract}

Background: Mycoplasmal pneumonia is an important infectious disease that threatens sheep and goat production worldwide, and Mycoplasma ovipneumoniae is one of major etiological agent causing mycoplasmal pneumonia. Recombinase polymerase amplification (RPA) is an isothermal nucleic acid amplification technique, and RPA-based diagnostic assays have been described for the detection of different types of pathogens.

Results: The RPA assays using real-time fluorescence detection (real-time RPA) and lateral flow strip detection (LFS RPA) were developed to detect M. ovipneumoniae targeting a conserved region of the 16S rRNA gene. Real-time RPA was performed in a portable florescence scanner at $39^{\circ} \mathrm{C}$ for $20 \mathrm{~min}$. LFS RPA was performed in a portable metal bath incubator at $39{ }^{\circ} \mathrm{C}$ for $15 \mathrm{~min}$, and the amplicons were visualized with the naked eyes within $5 \mathrm{~min}$ on the lateral flow strip. Both assays were highly specific for M. ovipneumoniae, as there were no cross-reactions with other microorganisms tested, especially the pathogens involved in respiratory complex and other mycoplasmas frequently identified in ruminant $\mathrm{s}$. The limit of detection of LFS RPA assay was $1.0 \times 101$ copies per reaction using a recombinant plasmid containing target gene as template, which is 10 times lower than the limit of detection of the real-time RPA and real-time PCR assays. The RPA assays were further validated on 111 clinical sheep nasal swab and fresh lung samples, and M. ovipneumoniae DNA was detected in 29 samples in the real-time RPA, 31 samples in the LFS RPA and 32 samples in the real-time PCR assay. Compared to real-time PCR, the real-time RPA and LFS RPA showed diagnostic specificity of $100 \%$ and $98.73 \%$, diagnostic sensitivity of $90.63 \%$ and $93.75 \%$, and a kappa coefficient of 0.932 and 0.934 , respectively.

Conclusions: The developed real-time RPA and LFS RPA assays provide the attractive and promising tools for rapid, convenient and reliable detection of $\mathrm{M}$. ovipneumoniae, especially in resource-limited settings.

\section{Background}

Mycoplasma ovipneumoniae is one of the major pathogens that cause mycoplasma pneumonia in sheep, goats, and wild ruminants [1-5]. M. ovipneumoniaeassociated respiratory disease is characterized by cough, gasp, runny noses, progressive weight loss, pulmonary interstitial hyperplasia inflammation, and variable morbidity and mortality rates between flocks $[6,7]$. Moreover, upon M. ovipneumoniae infection, sheep and goats become susceptible to other common pathogens causing respiratory disease, such as Mannheimia haemolytica, Pasteurella multocida and Parainfluenza-3 virus [8, 9]. Since first confirmed in Australia in 1972, infections by M. ovipneumoniae have been an endemic problem worldwide and have caused severe economic losses to the sheep and goat industry [10-12]. Bacteriological culture of $M$. ovipneumoniae is currently the gold standard for diagnosis, however, the culture is cumbersome and time-consuming due to the fastidious nature of the bacterium as well as that the follows required species identification by biochemical or serological tests, which make the assay burdensome for the routine applications [13-15]. In addition, the bacterial isolation may be hampered by sample contamination and prior antibiotic treatments received by the diseased animals. Serological tests, such as ELISA, indirect hemagglutination assay, are the common and economic methods for $M$. ovipneumoniae herd surveillance [12, 16]. However, seroconversion to $M$. ovipneumoniae is often delayed after natural infection, which makes the serology less effective in detecting early-stages of infection in herds, and unsuitable for detecting acute mycoplasmal pneumonia in the field [9, 14]. It is an urgent need to develop a rapid and accurate method to detect $M$. ovipneumoniae. Different nucleic acid amplification-based methods have been described to be sensitive and specific for M. ovipneumoniae, i.e. PCR, real-time PCR, and loop-mediated isothermal amplification (LAMP) $[9,14,15]$. PCR assays require a well-equipped laboratory, expensive equipment and trained personnel, which limits their application in the under-equipped laboratories and the point-of-need (PON) diagnosis $[9,15]$. Compared to the PCR assays, the isothermal amplification methods have advantages regarding convenience to perform and minimal equipment requirement. A LAMP assay for the detection of M. ovipneumoniae has been described for low requirement of experimental conditions, however, the assay requires $60 \mathrm{~min}$ to complete the reaction [14].

Recombinase polymerase amplification (RPA), an isothermal DNA amplification technique, is rapid, reliable and considered to be a promising approach for PON diagnosis $[17,18]$. RPA-based diagnostic assays have been described for the detection of different pathogens from different clinical samples $[19,20]$. In this study, a real-time RPA assay using the exo probe and a LFS

Page $2 / 11$ 
RPA assay using the nfo probe combined with lateral flow strip were developed for rapid, specific and sensitive detection of $M$. ovipneumoniae. The performance of the assays was further assessed by collecting and detecting the clinical sheep nasal swab and lung samples.

\section{Results}

\section{Analytical specificity and sensitivity of the RPA assays}

Specific amplification was only observed with M. ovipneumoniae, and there was no cross-reactions of other pathogens tested in both real-time RPA and LFS RPA assays (Fig.1). Five independent reactions were repeated and similar results were observed, demonstrating the good repeatability of the assays.

The limit of detection of LFS RPA assay was $1.0 \times 10^{1}$ copies M. ovipneumoniae standard DNA per reaction (Fig.2A), and the LOD of real-time RPA was $1.0 \times 10^{2}$ copies per reaction (Fig.2B), which was same as that of the real-time PCR (data not shown). The real-time RPA assay was further performed eight times on the molecular standard, in which $1.0 \times 10^{7}-1.0 \times 10^{2}$ copies DNA molecules were detected in $8 / 8$ runs, $1.0 \times 10^{1}-1.0 \times 10^{0}, 0 / 8$, which demonstrated the good reproducibility (Fig. 3).

\subsection{Validation of the RPA assays on clinical samples}

Of the 111 clinical samples, 29 (26.12\%), 31 (27.93\%) and 32 (28.83\%) samples were positive for M. ovipneumoniae by the realtime RPA, LFS RPA and real-time PCR, respectively (Table 2). Compared to real-time PCR, the real-time RPA and LFS RPA assays showed diagnostic specificity (DSp) of $100 \%$ and $98.73 \%$, diagnostic sensitivity (DSe) of $90.63 \%$ and $93.75 \%$, positive predictive value (PPV) of $100 \%$ and $96.77 \%$, negative predictive value (NPV) of $96.34 \%$ and $97.5 \%$, and kappa value of 0.932 and 0.934 , respectively (Table 3). The real-time RPA and the LFS RPA demonstrated the comparable performance in detecting the 111 clinical samples. It took less than 20 min in the RPA assays to obtain the positive results, while it took 32 - 46 min in the real-time RT-PCR with the Ct values ranging from 20.77 to 36.52 .

\section{Discussion}

The two developed real-time RPA and LFS RPA assays for detection of M. ovipneumoniae demonstrated to be rapid, specific, sensitive, and easy to perform. Both RPA assays performed well at $39^{\circ} \mathrm{C}$ within 20 min, which is faster than other common nucleic acid amplification methods. The real-time RPA assay and LFS RPA assay were performed on the tube scanner Genie III and a metal bath incubator, respectively. These two pieces of equipment are portable, lightweight, easily carried and can be charged by battery for working a whole day. Moreover, RPA reagents are provided in the form of lyophilized powder and cold chain independent. Several studies demonstrated that RPA is tolerant to most of the PCR inhibitors [19, 21]. The above characteristics make the developed RPA assays ideal for the PON detection of M. ovipneumoniae, which is especially important for farms located in rural areas.

Previous studies had demonstrated the efficacy of the PCR and LAMP to detect 16S rRNA and other conserved regions of genomic DNA of M. ovipneumoniae in different clinical specimens, including the nasal swabs and lung samples $[4,9,14,15]$. In this study, the RPA primers and probes were also designed based on the 16S rRNA gene. To ensure that the target sequences were unique to M. ovipneumoniae, we screened the selected primers and probes in silico using the pattern searching tool function from the EMBOSS package against the genomes of the common mycoplasmas causing infections in ruminants [22]. We were unable to find complementary regions when allowing 1 or 5 sequence mismatches for the primer sequences. Furthermore, there was no mismatch in the reverse primers and probes in the M. ovipneumoniae strains available in Genbank, only one mismatch in the forward primers in two strains: 2013-12928-46 (MN028079) and NCTC10151 (LR215028.1). According to the above in silico analysis, the designed primers and probes fulfilled the specificity requirements [23]. In the specificity analysis, both the real-time RPA and LFS RPA only amplified the genomic DNA of M. ovipneumoniae, and no other mycoplasmas, bacteria and PPRV. Most importantly, M. capricolum subsp. capripneumoniae, the etiological agent of contagious caprine pleuropneumonia, was not amplified by the new developed RPA assays. Although the in silico sequence analysis support 
that all the $M$. ovipneumoniae strains are detectable, it should be further confirmed by testing more genomic DNA of different strains of M. ovipneumoniae.

The diagnostic performances of the developed RPA assays were evaluated and compared to a real-time PCR assay, which is considered to be the gold standard of molecular detection methods. Based on the data in this study, the performances of the real-time RPA and LFS RPA assays were comparable to the real-time PCR, while the RPA assays had the distinct advantages of rapidness. Furthermore, the developed real-time RPA was slightly weak in the detection of the clinical samples containing low amounts of M. ovipneumoniae, as three nasal samples were negative in real-time RPA assay while positive in real-time PCR with $\mathrm{Ct}$ values of $36.49,35.50$ and 36.52 . Although the above results are inspiring, the RPA assays need be further validated by testing of more M. ovipneumoniae DNA positive clinical samples.

\section{Conclusions}

In this study, we describe the development of the real-time RPA and LFS RPA assays for the simple, rapid and reliable detection of $M$. ovipneumoniae from the sheep nasal and lung samples. The developed RPA assays could be performed in field conditions without the need of any expensive equipment, and could also become a routine test for rapid and direct detection of $M$. ovipneumoniae in the farm.

\section{Methods}

\section{Bacteria, virus strains, clinical samples and DNA extraction}

Genomic DNA of M. ovipneumoniae (Y98) and genomic DNA or CDNA of a panel of pathogens involved in respiratory complex and other mycoplasmas frequently identified in ruminant were maintained in our laboratory and used in the study, which were the following 6 mycoplasmas, 3 non-mycoplasma bacteria and 1 virus: M. capricolum subsp. capripneumoniae (F38), M. mycoides subsp. capri (PG3), M. arginini (G230), M. agalactiae (PG2), M. bovis (PG45), M. flocculare (HB-XS3), Mannheimia haemolytica (F120G3), Klebsiella pneumoniae (F21W3), Pasteurella multocida (F91G3) and Peste des petits ruminants virus (Nigeria 75/1 vaccine strain). Four artificial constructs, pUC57-Mbovoculi, pUC57-Mleachii, pUC57-Mcc and pUC57-Mdispar, were also used in the study. The constructs contain the full 16S rRNA gene of M.bovoculi (1531bp), M.leachii (1524bp), M. capricolum subsp. capricolum (1466bp) and M.dispar (1475bp), which were synthesized artificially by Sangon Biotech (Shanghai, China) based on the reference sequences available in GenBank (Accession numbers: CP007154, NR_044773, NR_118796, NR_025182).

A total of 46 sheep clinical samples (30 nasal swabs and 16 fresh lungs) were collected in Baoding City, Hebei Province from October to November 2019. The nasal swabs were collected from the sheep with coughing symptom in Fangzhuang farm in Dingzhou County, Baoding City, and the sheep fresh lungs were obtained from Zhuanluzhen slaughter house in Tang County, Baoding City. The sheep nasal swabs and lung samples were treated and the total DNA was extracted as described previously [24]. Furthermore, 65 nucleic acid extracted from the clinically healthy sheep nasal swabs were kindly provided by Dr. Qingan Han from Hebei Animal Disease Prevention and Control Center. The 65 sheep nasal swabs were collected in October-December 2019, in which 35 samples were collected from one sheep farm in Tang Country, Baoding City and the other 30 samples were collected from one sheep farm in Pingquan County, Chengde City. All the samples were used for the daily sheep disease surveillance. All DNA were quantified using a ND-2000c spectrophotometer (NanoDrop, Wilmington, USA) and stored at $-80{ }^{\circ} \mathrm{C}$ until use.

\section{Generation of standard DNA}

To generate a M. ovipneumoniae standard DNA for the RPA assays, a PCR product containing $361 \mathrm{bp}$ covering the region of interest of 16S rRNA gene was amplified from the M. ovipneumoniae DNA using LMF1 and LMR1 as primers (Table 1) and cloned into the pMD19-T (Takara, Dalian, China) for standards. The resulting plasmid, pMO-16SrRNA, was transformed into Escherichia coli DH5a cells, purified with the SanPrep Plasmid MiniPrep Kit (Sangon Biotech, Shanghai, China) and quantified using a ND-2000c spectrophotometer. The copy number of DNA molecules was calculated by the following formula: amount 
$($ copies $/ \mu \mathrm{L})=[\mathrm{DNA}$ concentration $(\mathrm{g} / \mu \mathrm{L}) /($ plasmid length in base pairs $\times 660)] \times 6.02 \times 10^{23}$. Ten-fold dilutions of the pMO16SrRNA, ranging from $1.0 \times 10^{7}$ to $1.0 \times 10^{0} \mathrm{copies} / \mu \mathrm{L}$, were prepared in nuclease-free water and aliquots of each dilution were stored at $-80 \circ \mathrm{C}$.

\section{RPA primers and probe}

Nucleotide sequence data for different $M$. ovipneumoniae strains available in GenBank were aligned to identify the conserved regions in the 16S rRNA gene, which was determined as the molecular target for RPA. According to the reference sequences of M. ovipneumoniae (Accession numbers: NR_025989.1, LR215028.1, MN028361, MN028184, MN028079, MH133233), the RPA primers, exo and nfo probes were designed following the RPA manufacturer guidelines (TwistDx. Cambridge, UK). Primers and probe are listed in Table 1 and were synthesized by Sangon Biotech, Shanghai, China.

\section{Real-time RPA and LFS RPA assays}

The M. ovipneumoniae real-time RPA assay was performed as described previously [24]. The total reaction volume was $50 \mu \mathrm{L}$ including $40.9 \mu \mathrm{L}$ of Buffer A (rehydration buffer), $2.0 \mu \mathrm{L}$ of each RPA primers (MO-exo-F and MO-exo-R, $10 \mu \mathrm{mol} / \mathrm{L}$ ), $0.6 \mu \mathrm{L}$ of exo probe (MO-exo-P, $10 \mu \mathrm{mol} / \mathrm{L}$ ) and $2.5 \mu \mathrm{L}$ of Buffer B (magnesium acetate, $280 \mathrm{mmol} / \mathrm{L}$ ). Furthermore, $1 \mu \mathrm{L}$ of genomic DNA or recombinant plasmid was used for the specificity and sensitivity analysis, or $2 \mu \mathrm{L}$ of sample DNA was used for the clinical sample diagnosis.

The M. ovipneumoniae LFS RPA assay were performed as described previously [24]. The total reaction volume was $50 \mu \mathrm{L}$ including $29.5 \mu \mathrm{L}$ of rehydration buffer, $2.1 \mu \mathrm{L}$ of each RPA primers (MO-nfo-F and MO-nfo-R, $10 \mu \mathrm{mol} / \mathrm{L}$ ), $0.6 \mu \mathrm{L}$ of exo probe (MO-nfo-P, $10 \mu \mathrm{mol} / \mathrm{L})$ and $2.5 \mu \mathrm{L}$ of magnesium acetate $(280 \mathrm{mmol} / \mathrm{L})$. In addition, $1 \mu \mathrm{L}$ of bacterial genomic DNA or recombinant plasmid was used for the specific and sensitive analysis, or $2 \mu \mathrm{L}$ of sample DNA was used for the clinical sample diagnosis. The assay was performed in a metal bath incubator at $39{ }^{\circ} \mathrm{C}$ for $15 \mathrm{~min}$. Furthermore, the lateral flow strips (Milenia Biotec $\mathrm{GmbH}$, Germany) were used to detect the RPA amplicons dual-labeled with FAM and biotin.

\section{Analytical specificity and sensitivity analysis}

Both RPA assays were performed to amplify the nucleic acids of a panel of microorganisms including M. ovipneumoniae, $M$. capricolum subsp. capripneumoniae, M. mycoides subsp. capricolum, M. arginini, M. agalactiae, M.bovoculi, M.leachii, M. capricolum subsp. capricolum, M.dispar, M. bovis, M. flocculare, M. haemolytica, P. multocida, K. pneumoniae, PPRV, which are considered to be dangerous to the sheep and goat respiratory system or frequently identified in the ruminants. The analytical specificity analysis was repeated five times.

The standard DNA of M. ovipneumoniae, ranging from $1.0 \times 10^{7}$ to $1.0 \times 10^{0} \mathrm{copies} / \mu \mathrm{L}$, was prepared in nuclease-free water and used for the RPA analytical sensitivity analysis. One microliter of each dilution was amplified by both RPA assays to determine the limit of detection (LOD). The analytical sensitivity analysis was repeated five times. Furthermore, the real-time RPA was tested using the standard DNA in 8 replicates, the threshold time was plotted against the molecules detected and a semi-log regression was calculated using Prism software 5.0 (Graphpad Software Inc., SanDiego, California).

\section{Validation with clinical samples}

The real-time RPA assay was assessed with 95 sheep nasal swabs and 16 sheep fresh lungs. All samples tested with the two RPA assays were also tested by a real-time PCR in parallel. The real-time PCR for M. oviopneumoniae was performed on a ABI 7500 instrument (Applied Biosystems, Foster City, California) described previously [4].

\section{Abbreviations}

CT: Cycle threshold; DSp: diagnostic specificity; DSe: diagnostic sensitivity; ELISA: enzyme linked immunosorbent assay; K. pneumonia: Klebsiella pneumoniae; LAMP: loop-mediated isothermal amplification; LFS: lateral flow strip; M. agalactiae: Mycoplasma agalactiae; M. arginini: Mycoplasma arginini; M. bovis: Mycoplasma bovis; M.bovoculi: Mycoplasma bovoculi; M. 
capricolum subsp. capripneumoniae: Mycoplasma capricolum subsp. capripneumoniae; M.dispar: Mycoplasma dispar; M. flocculare: Mycoplasma flocculare; M. haemolytica: Mannheimia haemolytica; M.leachii: Mycoplasma leachii; M. mycoides subsp. capri: Mycoplasma mycoides subsp. capri; M. ovipneumoniae: Mycoplasma ovipneumoniae; NPV: negative predictive value; PCR: polymerase chain reaction; P. multocida: Pasteurella multocida; PON: Point-of-need; PPRV: Peste des petits ruminants virus; PPV: positive predictive value; RPA: Recombinase polymerase amplification; TT: Threshold time.

\section{Declarations}

\section{Ethics approval and consent to participate}

The sheep nasal swabs and sheep fresh lungs used in this study were collected in sheep husbandry farms and a slaughter house, respectively. The written consents for the use of the samples before participation in the study were obtained from the farmers and the slaughter house's owner. This study was approved by the Institutional Animal Care and Ethics Committee of Hebei Agricultural University (approval no. IACECHEBAU20110509).

\section{Consent for publication}

Not applicable.

\section{Availability of data and materials}

The dataset analyzed during the current study is available from the corresponding author on reasonable request.

\section{Competing interests}

The authors declare that they have no competing interests.

\section{Funding}

This work was supported by the Project for Key Common Technologies for High Quality Agricultural Development of Hebei Province (19226636D), and the Earmarked Fund for Hebei Sheep and Goat Innovation Team of Modern Agro-industry Technology Research System (HBCT2018140204). The funding agencies had no role in study design; in the collection, analysis and interpretation of data; in the writing of the report; or in the decision to submit the article for publication.

\section{Authors' contributions}

JCW and WZY conceived and designed the study. JFW and RWL developed the real-time RPA and LFS RPA assays and analyzed the data. XXS, LBL and XPH performed the clinical samples testing, helped in the data analysis and manuscript revision. JCW and WZY wrote the manuscript. All authors read and approved the final manuscript.

\section{Acknowledgements}

The authors thank the laboratory staff in the animal hospital of Hebei Agricultural University. We also thank Dr. Qingan Han from the Hebei Animal Disease Prevention and Control Center for providing the 65 nucleic acid samples.

\section{Authors' information}

1Technology Center of Shijiazhuang Customs District, Shijiazhuang 050051, China

2College of Veterinary Medicine, Hebei Agricultural University, Baoding 071001, China

3Hebei Academy of Science and Technology for Inspection and Quarantine, Shijiazhuang 050051, China

\section{References}


1. Alley MR, lonas G, Clarke JK: Chronic non-progressive pneumonia of sheep in New Zealand - a review of the role of Mycoplasma ovipneumoniae. New Zealand veterinary journal 1999, 47(5):155-160.

2. Highland MA, Herndon DR, Bender SC, Hansen L, Gerlach RF, Beckmen KB: Mycoplasma ovipneumoniae in Wildlife Species beyond Subfamily Caprinae. Emerging infectious diseases 2018, 24(12):2384-2386.

3. Mohan K, Obwolo MJ, Hill FW: Mycoplasma ovipneumoniae infection in Zimbabwean goats and sheep. Journal of comparative pathology 1992, 107(1):73-79.

4. Handeland K, Tengs T, Kokotovic B, Vikoren T, Ayling RD, Bergsjo B, Sigurethardottir OG, Bretten T: Mycoplasma ovipneumoniae-a primary cause of severe pneumonia epizootics in the Norwegian Muskox (Ovibos moschatus) population. PloS one 2014, 9(9):e106116.

5. DaMassa AJ, Wakenell PS, Brooks DL: Mycoplasmas of goats and sheep. Journal of veterinary diagnostic investigation : official publication of the American Association of Veterinary Laboratory Diagnosticians, Inc 1992, 4(1):101-113.

6. Besser TE, Cassirer EF, Potter KA, VanderSchalie J, Fischer A, Knowles DP, Herndon DR, Rurangirwa FR, Weiser GC, Srikumaran S: Association of Mycoplasma ovipneumoniae infection with population-limiting respiratory disease in freeranging Rocky Mountain bighorn sheep (Ovis canadensis canadensis). Journal of clinical microbiology 2008, 46(2):423430.

7. Rifatbegovic M, Maksimovic Z, Hulaj B: Mycoplasma ovipneumoniae associated with severe respiratory disease in goats. The Veterinary record 2011, 168(21):565.

8. Sheehan M, Cassidy JP, Brady J, Ball H, Doherty ML, Quinn PJ, Nicholas RA, Markey BK: An aetiopathological study of chronic bronchopneumonia in lambs in Ireland. Veterinary journal 2007, 173(3):630-637.

9. McAuliffe L, Hatchell FM, Ayling RD, King Al, Nicholas RA: Detection of Mycoplasma ovipneumoniae in Pasteurellavaccinated sheep flocks with respiratory disease in England. The Veterinary record 2003, 153(22):687-688.

10. Carmichael LE, St George TD, Sullivan ND, Horsfall N: Isolation, propagation, and characterization studies of an ovine Mycoplasma responsible for proliferative interstitial pneumonia. The Cornell veterinarian 1972, 62(4):654-679.

11. Giangaspero M, Nicholas RA, Hlusek M, Bonfini B, Osawa T, Orusa R, Tatami S, Takagi E, Moriya H, Okura N et al: Seroepidemiological survey of sheep flocks from Northern Japan for Mycoplasma ovipneumoniae and Mycoplasma agalactiae. Tropical animal health and production 2012, 44(3):395-398.

12. Cheng C, Jun Q, Qingling M, Zhengxiang H, Yu M, Xuepeng C, Zibing C, Jinsheng Z, Zaichao Z, Kuojun C et al: Serological and molecular survey of sheep infected with Mycoplasma ovipneumoniae in Xinjiang, China. Tropical animal health and production 2015, 47(8):1641-1647.

13. Weiser GC, Drew ML, Cassirer EF, Ward AC: Detection of Mycoplasma ovipneumoniae and M. arginini in bighorn sheep using enrichment culture coupled with genus- and species-specific polymerase chain reaction. Journal of wildlife diseases 2012, 48(2):449-453.

14. Zhang J, Cao J, Zhu M, Xu M, Shi F: Loop-mediated isothermal amplification-lateral-flow dipstick (LAMP-LFD) to detect Mycoplasma ovipneumoniae. World journal of microbiology \& biotechnology 2019, 35(2):31.

15. Yang F, Dao X, Rodriguez-Palacios A, Feng X, Tang C, Yang X, Yue H: A real-time PCR for detection and quantification of Mycoplasma ovipneumoniae. The Journal of veterinary medical science 2014, 76(12):1631-1634.

16. Rong G, Zhao JM, Hou GY, Zhou HL: Seroprevalence and molecular detection of Mycoplasma ovipneumoniae in goats in tropical China. Tropical animal health and production 2014, 46(8):1491-1495.

17. Piepenburg O, Williams CH, Stemple DL, Armes NA: DNA detection using recombination proteins. PLoS biology 2006, 4(7):e204.

18. Amer HM, Abd El Wahed A, Shalaby MA, Almajhdi FN, Hufert FT, Weidmann M: A new approach for diagnosis of bovine coronavirus using a reverse transcription recombinase polymerase amplification assay. Journal of virological methods 2013, 193(2):337-340.

19. Daher RK, Stewart G, Boissinot M, Bergeron MG: Recombinase Polymerase Amplification for Diagnostic Applications. Clinical chemistry 2016, 62(7):947-958. 
20. Li J, Macdonald J, von Stetten F: Review: a comprehensive summary of a decade development of the recombinase polymerase amplification. The Analyst 2018, 144(1):31-67.

21. Lillis L, Siverson J, Lee A, Cantera J, Parker M, Piepenburg O, Lehman DA, Boyle DS: Factors influencing Recombinase polymerase amplification (RPA) assay outcomes at point of care. Molecular and cellular probes 2016, 30(2):74-78.

22. Rice P, Longden I, Bleasby A: EMBOSS: the European Molecular Biology Open Software Suite. Trends in genetics : TIG 2000, 16(6):276-277.

23. Daher RK, Stewart G, Boissinot M, Boudreau DK, Bergeron MG: Influence of sequence mismatches on the specificity of recombinase polymerase amplification technology. Molecular and cellular probes 2015, 29(2):116-121.

24. Liu L, Li R, Zhang R, Wang J, An Q, Han Q, Wang J, Yuan W: Rapid and sensitive detection of Mycoplasma hyopneumoniae by recombinase polymerase amplification assay. Journal of microbiological methods 2019, 159:56-61.

\section{Tables}

Table 1 Sequences of the primers and probes for M .ovipneumoniae real-time RPA, LFS RPA and PCR assays

\begin{tabular}{|c|c|c|c|c|}
\hline Assay & $\begin{array}{l}\text { Primers and } \\
\text { probes }\end{array}$ & Sequence $5^{\prime}-3^{\prime}$ & $\begin{array}{l}\text { Amplicon } \\
\text { size (bp) }\end{array}$ & References \\
\hline \multirow{3}{*}{$\begin{array}{l}\text { real- } \\
\text { time } \\
\text { RPA }\end{array}$} & MO-exo-F & TGAGTAACACGTACCTAACCTACCTTTTGGAC & \multirow[t]{4}{*}{254} & \multirow[t]{4}{*}{ This study } \\
\hline & MO-exo-R & TGCTGCCTCCCGTAGGAGTCTGGGCCGTATCTC & & \\
\hline & MO-exo-P & $\begin{array}{l}\text { TTGGTAGGGTAAAGGCCTACCAAGACGATGA (FAM-dT)(THF)(BHQ1- } \\
\text { dT)TTAGCGGGGCCAAGAG }\end{array}$ & & \\
\hline \multirow{4}{*}{$\begin{array}{l}\text { LFS } \\
\text { RPA }\end{array}$} & & -C3-spacer & & \\
\hline & MO-nfo-F & TGAGTAACACGTACCTAACCTACCTTTTGGAC & \multirow[t]{3}{*}{254} & \multirow[t]{3}{*}{ This study } \\
\hline & MO-nfo-R & Biotin-TGCTGCCTCCCGTAGGAGTCTGGGCCGTATCTC & & \\
\hline & MO-nfo-P & $\begin{array}{l}\text { FAM- } \\
\text { TTGGTAGGGTAAAGGCCTACCAAGACGATGAT(THF)TTTAGCGGGGCCAAGAG- } \\
\text { C3-spacer }\end{array}$ & & \\
\hline real- & Mo16S_35F & TGGGTGAGTAACACGTACCTAACC & 62 & [4] \\
\hline time & Mo16S_96R & AGCCGCTGTTTCCAATGG & & \\
\hline PCR & Mo16S_60T & FAM-ACCTTTTGGACCGGGATA-MGB & & \\
\hline \multirow{2}{*}{ PCR } & LMF1 & TGAACGGAATATGTTAGCTT & \multirow[t]{2}{*}{361} & \multirow[t]{2}{*}{ [9] } \\
\hline & LMR1 & GACTTCATCCTGCACTCTGT & & \\
\hline
\end{tabular}

Table 2 Comparison of M. ovipneumoniae real-time RPA, LFS RPA and real-time PCR assays for detection of clinical samples

\begin{tabular}{|c|c|c|c|c|c|c|c|c|c|}
\hline \multirow[t]{2}{*}{ Origin } & \multirow[t]{2}{*}{ Location } & \multirow[t]{2}{*}{ Sample } & \multirow[t]{2}{*}{ Number } & \multicolumn{2}{|c|}{ Real-time RPA } & \multicolumn{2}{|c|}{ LFS RPA } & \multicolumn{2}{|c|}{ Real-time PCR } \\
\hline & & & & $\mathrm{P}$ & $\mathrm{N}$ & $P$ & $\mathrm{~N}$ & $P$ & $\mathrm{~N}$ \\
\hline Farm 1 & Dingzhou County, Baoding & nasal swabs & 30 & 11 & 19 & 11 & 19 & 13 & 17 \\
\hline \multirow[t]{2}{*}{ Farm 2} & Tang County, Baoding & nasal & 35 & 4 & 31 & 5 & 30 & 5 & 30 \\
\hline & & swabs & & & & & & & \\
\hline Farm 3 & Pingquan County, Chengde & nasal swabs & 30 & 8 & 22 & 9 & 21 & 8 & 22 \\
\hline \multirow[t]{2}{*}{ Slaughter house } & Tang County, Baoding & fresh lungs & 16 & 6 & 10 & 6 & 10 & 6 & 10 \\
\hline & & $\mathrm{T}$ & 111 & 29 & 82 & 31 & 80 & 32 & 79 \\
\hline
\end{tabular}


Table 3 Diagnostic sensitivity, diagnostic specificity, predictive value, and kappa value of real-time RPA, LFS RPA and real-time PCR assays for diagnosing M. ovipneumoniae infection

\begin{tabular}{|c|c|c|c|c|}
\hline \multicolumn{5}{|c|}{ real-time PCR } \\
\hline & & $\mathrm{P}$ & $\mathrm{N}$ & $\mathrm{T}$ \\
\hline \multirow[t]{5}{*}{ real-time RPA } & $\mathrm{P}$ & 29 & 0 & 29 \\
\hline & $\mathrm{N}$ & 3 & 79 & 82 \\
\hline & $\mathrm{T}$ & 32 & 79 & 111 \\
\hline & & DSe:90.63\% & DSp:100\% & $\mathrm{K}: 0.932$ \\
\hline & & PPV:100\% & NPV:96.34\% & \\
\hline \multirow[t]{5}{*}{ LFS RPA } & $\mathrm{P}$ & 30 & 1 & 31 \\
\hline & $\mathrm{N}$ & 2 & 78 & 80 \\
\hline & $\mathrm{T}$ & 32 & 79 & 111 \\
\hline & & DSe:93.75\% & DSp:98.73\% & $\mathrm{K}: 0.934$ \\
\hline & & PPV:96.77\% & NPV:97.5\% & \\
\hline
\end{tabular}

Note: P, positive; N, negative; DSe, diagnostic sensitivity; DSp: diagnostic specificity; K: kappa value; PPV: positive predictive value; NPV: negative predictive value

\section{Figures}
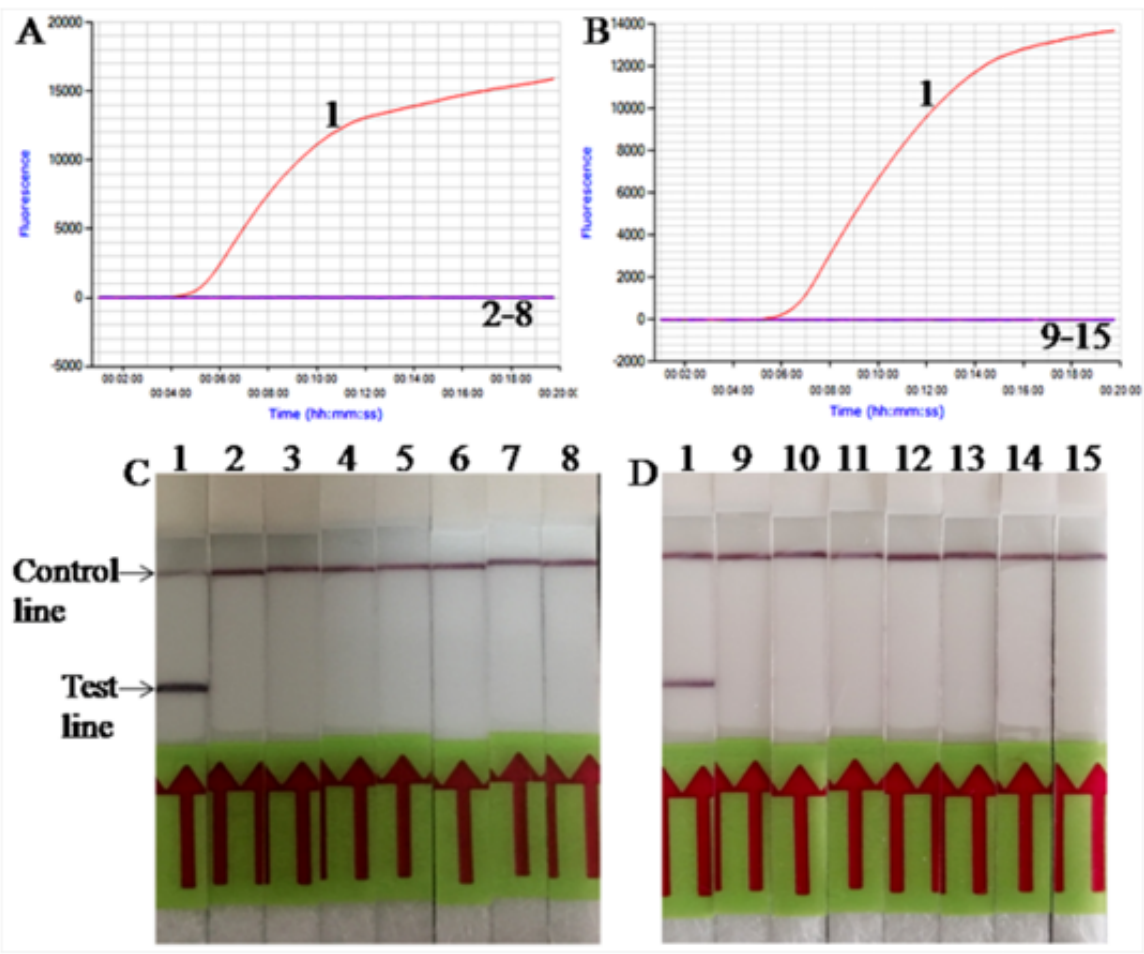

Figure 1 
Analytical Specificity of M. ovipneumoniae real-time RPA (A, B) and LFS RPA (C, D) assays. Only the M. ovipneumoniae was amplified, but not other pathogens tested $(n=5)$. lane 1, M. ovipneumoniae; lane 2, M. capricolum subsp. capripneumoniae; lane 3, M. mycoides subsp. capri; lane 4, M. arginini; lane 5, M. agalactiae; lane 6, P. multocida; lane 7, K. pneumoniae; lane 8, PPRV; lane 9, M. bovis; lane 10, M. flocculare; lane 11, M.bovoculi; lane 12, M.leachii; lane 13, M. capricolum subsp. capricolum; lane 14, M.dispar; lane 15, M. haemolytica.
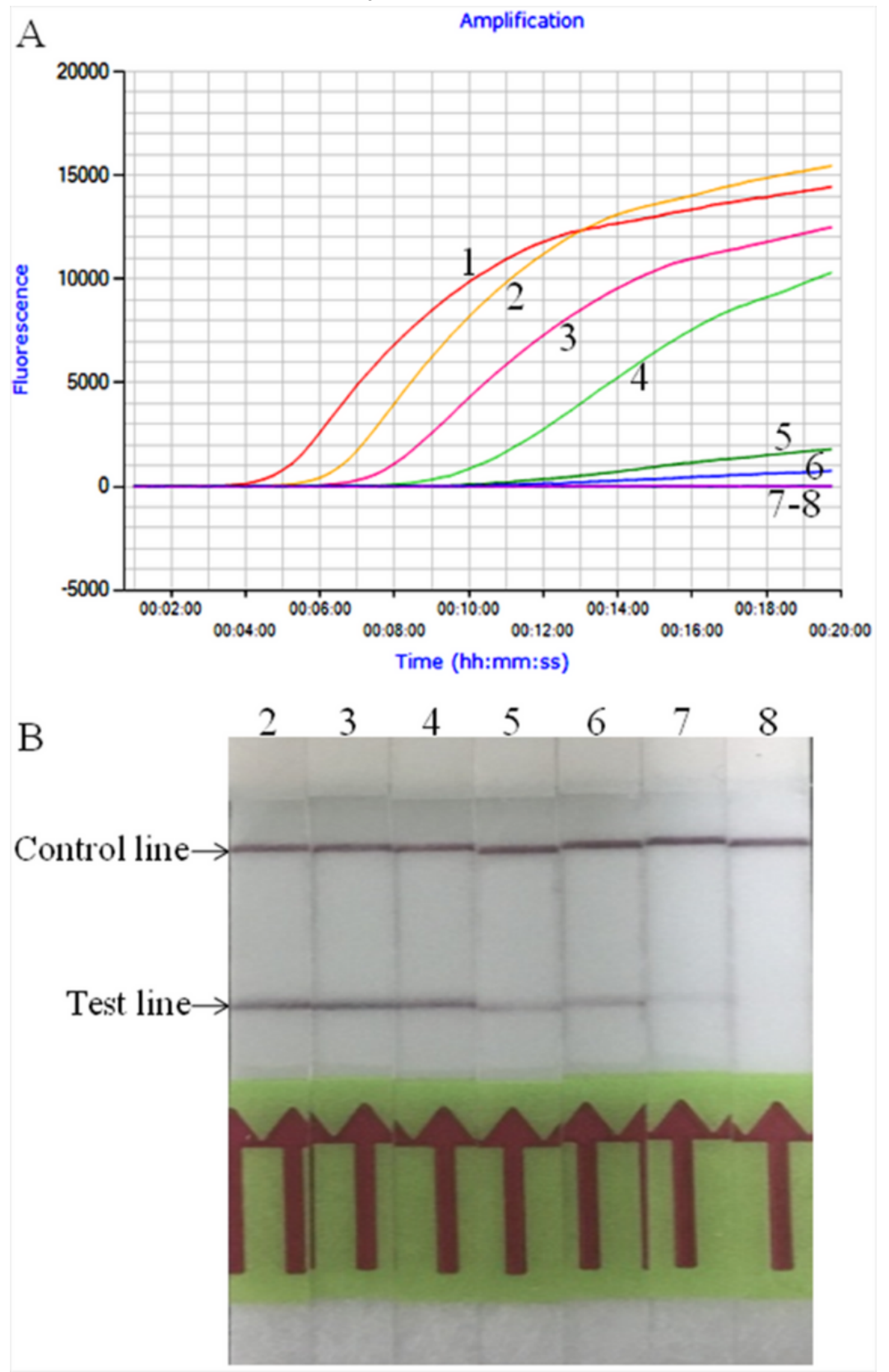

\section{Figure 2}

Analytical Sensitivity of M. ovipneumoniae real-time RPA (A) and LFS RPA (B) assays. The LOD of the real-time RPA was $1.0 \times 102$ copies per reaction of M. ovipneumoniae standard DNA, while the LOD of the LFS RPA was 1.0×101 copies per reaction. lane 1, 1.0×107 copies; lane 2, 1.0×106 copies; lane 3, 1.0×105 copies; lane 4, 1.0×104 copies; lane 5, 1.0×103 copies; lane 6, $1.0 \times 102$ copies; lane 7, 1.0×101 copies; lane 8, 1.0×100 copies. 


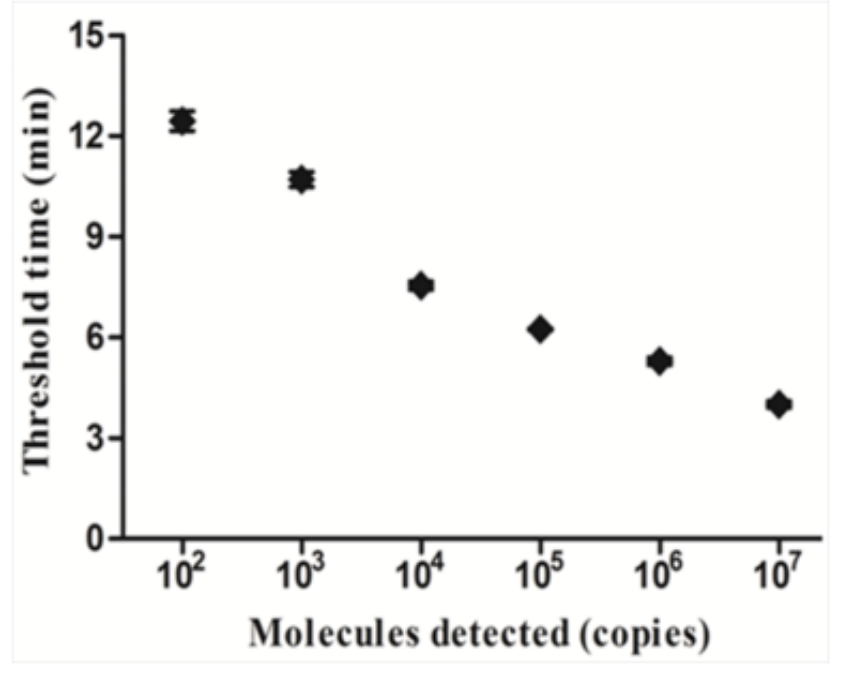

\section{Figure 3}

Reproducibility of M. ovipneumoniae real-time RPA assay. The analytical sensitivity was determined on DNA molecular standard (8 runs) for real-time RPA. Semi-logarithmic regression of the data collected from real-time RPA test runs on the DNA molecular standards using Prism Software. The run time of the real-time RPA was between 4 min-13 min for 1.0 × 107-1.0 × 102 copies M. ovipneumoniae standard DNA. 